\title{
Facial Nerve Disorder, CTCAE
}

National Cancer Institute

\section{Source}

National Cancer Institute. Facial Nerve Disorder, CT CAE. NCI Thesaurus. Code C143473.

A disorder characterized by dysfunction of the facial nerve (seventh cranial nerve). 